\title{
Iteration Algebras EXTENDED ABSTRACT
}

\author{
Stephen L. Bloom \\ Stevens Institute of Technology \\ Department of Computer Science \\ Hoboken, $\mathrm{NJ} 07030$
}

\author{
Zoltán Ésik* \\ A. Jozsef University \\ Bolyai Institute \\ Szeged, Hungary
}

There is an ad hoc syntactic notion involved in the basic notions of standard universal algebra: that of a signature. We review how syntax was removed by Lawvere's introduction [Law63] of the notion of an algebraic theory.

Let $\Sigma$ be a signature. A $\Sigma$-algebra $\mathfrak{A}$ consists of a set $A$ and a function $f_{\mathfrak{x}}: A^{n} \rightarrow A$ for each letter $f$ in $\Sigma$ of rank $n, n \geq 0$. We assume that the notion of a $\Sigma$-term built from variables in the set $\left\{x_{1}, \ldots, x_{n}\right\}$ is familiar. Each $\Sigma$-term $t$ built from $\left\{x_{1}, \ldots, x_{n}\right\}$ determines a function

$$
t_{\mathfrak{a}}: A^{n} \rightarrow A \text {. }
$$

Two such terms $t, t^{t}$ are equivalent in $\mathfrak{A}$ if the functions $t_{\mathfrak{a}}$ and $t_{\mathfrak{a}}^{\prime}$ are the same. The structure of equivalence classes of terms under $\mathfrak{A}$-equivalence, for all algebras $\mathfrak{A}$ in a class $K$ was analyzed by Lawvere [Law63] as an algebraic theory. Briefly, an algebraic theory $T$ is a category whose objects are the nonnegative integers and in which $n$ is the copower of 1 with itself $n$-times. One class of algebraic theories is the following. For each set $A$, the algebraic theory Pow $_{A}$ has all functions $A^{p} \rightarrow A^{n}$ as morphisms $n \rightarrow p$; copower morphisms $i_{n}: 1 \rightarrow n, i \in[n]$, are the projection functions $A^{n} \rightarrow A$. If $T$ is an algebraic theory, a $T$-algebra $(A, \alpha)$ is a functor $\alpha: T \rightarrow$ Pow $_{A}$ which preserves objects and the copower injections. Thus, if $f: n \rightarrow p$ in $T, n \alpha \stackrel{f_{\alpha}}{\rightarrow} p \alpha$ in Pow $A$; i.e.

$$
A^{p} \stackrel{f_{\alpha}}{\rightarrow} A^{n}
$$

A morphism $(A, \alpha) \rightarrow(B, \beta)$ of $T$-algebras (i.e. a homomorphism) is a natural transformation $h: \alpha \rightarrow$ $\beta$. It can be shown that for any fixed theory $T$, the collection of all $T$-algebras and morphisms is a category which is isomorphic to a variety of $\Sigma$-algebras for several possible choices of $\Sigma$. Conversely, any signature $\Sigma$, each variety $K$ of $\Sigma$-algebras determines an algebraic theory $T$ such that $K$ is isomorphic to the category of all $T$-algebras. In this framework, the role of $\Sigma$-terms on the variables $\left\{x_{1}, \ldots, x_{n}\right\}$ is played by the morphisms $1 \rightarrow n$ in the theory. Thus, in particular, there is one algebraic theory $T$ such that the category of $T$-algebras is isomorphic to the variety of all groups. No particular choice of a signature for groups is required; all equivalent choices yield the same algebraic theory.

In several papers (see [BEW80a, BEW80b, Ési80, BÉ88] and the references there), properties of algebraic theories enriched with a fixed point operation

$$
f \rightarrow f^{\dagger}
$$

satisfying certain identities have been studied. These theories, iteration theories, seem to capture all of the equational properties of iterative processes, whatever they are. As samples, the inputoutput behaviors and the stepwise behaviors of flowchart algorithms, the matrices of regular sets, the bisimulation equivalence classes of synchronization trees all form iteration theories.

\footnotetext{
"Partially supported by an Alexander von Humboldt Fellowship
} 
In the current paper, we are interested in the algebras of iteration theories. It is a fact, noticed in particular in Wagner [Wag90], that in many theoretical studies of iteration, theories have played the central role, and not their algebras. This phenomenon is perhaps due to the fact that little effort has been devoted to studying these algebras, except in special cases. As examples, we mention the ordered $\omega$-continuous algebras [WTGW76, GTWW77, Niv75, CN76, Guess81] and the iterative and regular algebras [Nel81, Tiu78].

If $\mathfrak{A}=(A, \alpha)$ is an iteration algebra, i.e. an algebra of some iteration theory $T$ (see the next section for a precise definition), the functions $t_{\alpha}: A^{1+p} \rightarrow A$ induced by the $T$-morphisms $t: 1 \rightarrow 1+p$ in $T$ all have the following fixed point property.

$$
\forall y \in A^{p} \exists x \in A\left[x=t_{\alpha}(x, y)\right]
$$

More generally, for any $n$-tuple of functions $\left(t_{i}\right)_{\alpha}: A^{n+p} \rightarrow A$ induced by $T$-morphisms $t_{i}: 1 \rightarrow n+p$,

$$
\forall y \in A^{p} \exists x_{1}, \ldots x_{n} \in A\left[x_{i}=\left(t_{i}\right)_{\alpha}\left(x_{1}, \ldots, x_{n}, y\right)\right],
$$

for each $i \in[n]$. Thus, in algebras of iteration theories, there are solutions for certain systems of fixed point equations. The reason that solutions exist is that in each iteration theory, for any morphism $t: n \rightarrow n+p$, the morphism $t^{\dagger}$ satisfies the fixed point identity $t^{\dagger}=t \cdot\left\langle t^{\dagger}, 1_{p}\right\rangle$. Hence, for any $y \in A^{p}$,

$$
\left(t^{\dagger}\right)_{\alpha}(y)=t_{\alpha}\left(\left(t^{\dagger}\right)_{\alpha}(y), y\right) .
$$

Thus, the morphisms $t^{t}$ determine canonical solutions for the fixed point equation for $t$ :

$$
x=t_{\alpha}(x, y) \text {. }
$$

What is meant by canonical solutions? That the axioms for iteration theories are satisfied.

We discuss the meaning of three of the four axioms in [Ési80] in the context of theories functions.

Suppose that $f: A^{p} \rightarrow A^{n}$. Define the function $\left(0_{n} \oplus f\right): A^{n+p} \rightarrow A^{n}$ by

$$
\left(0_{n} \oplus f\right)(\bar{x}, \bar{y})=f(\bar{y})
$$

The fixed point identity forces the definition:

$$
\begin{aligned}
\left(0_{n} \oplus f\right)^{\dagger}: A^{p} & \rightarrow A^{n} \\
\bar{y} & \mapsto f(\bar{y}) .
\end{aligned}
$$

Thus, $\left(0_{n} \oplus f\right)^{\dagger}=f$. This equation is one of a possible set of axioms for iteration theories.

Now for any function $g: A^{k} \rightarrow A^{r}$ and any $q \geq 0$, define the function $g \oplus 0_{q}: A^{k+q} \rightarrow A^{r}$ by

$$
\left(g \oplus 0_{q}\right)(\bar{y}, \bar{z})=g(\bar{y}) .
$$

When $f: A^{n+p} \rightarrow A^{n}$, the fixed point equation for $\left(f \oplus 0_{q}\right): A^{n+p+q} \rightarrow A^{n}$ is $\bar{x}=\left(f \oplus 0_{q}\right)(\bar{x}, \bar{y}, \bar{z})$, i.e. $\bar{x}=f(\bar{x}, \bar{y})$. It is natural then, to define

$$
\left(f \oplus 0_{q}\right)^{\dagger}=f^{\dagger} \oplus 0_{q}
$$

which is the second axiom in [Ési80].

Lastly, suppose we are given functions

$$
\begin{aligned}
& f_{1}: A^{n+m+p} \rightarrow A^{n} \\
& f_{2}: A^{n+m+p} \rightarrow A^{m} .
\end{aligned}
$$


By target tupling these functions we obtain the function

$$
\begin{aligned}
\left\langle f_{1}, f_{2}\right\rangle: A^{n+m+p} & \rightarrow A^{n+m} \\
(\bar{x}, \bar{y}, \bar{z}) & \mapsto\left(f_{1}(\bar{x}, \bar{y}, \bar{z}), f_{2}(\bar{x}, \bar{y}, \bar{z})\right) .
\end{aligned}
$$

Consider the system of fixed point equations:

$$
(\bar{x}, \bar{y})=\left(f_{1}(\bar{x}, \bar{y}, \bar{z}), f_{2}(\bar{x}, \bar{y}, \bar{z})\right) .
$$

If we solve these fixed point equations one at a time, we obtain first $\bar{x}=f_{1}^{\dagger}(\bar{y}, \bar{z})$. Then, substitute this value for $\bar{x}$ in the second equation and solve for the $\bar{y}$ in the equation

$$
\bar{y}=h(\bar{y}, \bar{z})
$$

where $h$ is the function $A^{m+p} \rightarrow A^{m}$ defined by:

$$
h(\bar{y}, \bar{z})=f_{2}\left(f_{1}^{\dagger}(\bar{y}, \bar{z}), \bar{y}, \bar{z}\right) .
$$

The solution is $\bar{y}=h^{\dagger}(\bar{z})$. Thus, we have the pairing identity for $\left\langle f_{1}, f_{2}\right\rangle^{\dagger}: A^{p} \rightarrow A^{n+m}$ :

$$
\begin{aligned}
\left\langle f_{1}, f_{2}\right\rangle^{\dagger} & =\left(f_{1}^{\dagger} \cdot\left\langle h^{\dagger}, \mathbf{1}_{p}\right\rangle, h^{\dagger}\right\rangle \\
\bar{z} & \mapsto\left(f_{1}^{\dagger}\left(h^{\dagger}(\bar{z}), \bar{z}\right), h^{\dagger}(\bar{z})\right) .
\end{aligned}
$$

There is one other axiom whose justification is less obvious, and we omit it. The point of the above discussion is to show that iteration algebras arise naturally when one is interested in solving systems of fixed point equations in structures having enough polynomial functions to serve as Skolem functions.

The category of all iteration algebras of an iteration theory $T$ forms a variety of iteration algebras. In more familiar terms, a variety of iteration algebras is a collection of algebras which are models for a set of first order sentences. These sentences can be divided into a set of equations and a set of compatibility conditions. Some of the equations follow from identities valid in all iteration theories, such as

$$
t^{\dagger}(\bar{y})=t\left(t^{\dagger}(\bar{y}), \bar{y}\right)
$$

for each term $t(\bar{x}, \bar{y})$; others are valid only in a given theory $T$, e.g.

$$
t(x, x)=x,
$$

for some term $t(x, y)$. The compatibility conditions ensure that whenever two terms $t, u$ are interpreted as the same function, then the terms $t^{\dagger}$ and $u^{\dagger}$ are also interpreted as the same function.

The authors had hoped that all of the familiar properties of standard varieties would hold also for varieties of iteration algebras. Indeed, our first result was a Birkhoff type theorem: a class $\mathcal{V}$ of iteration algebras is a variety iff $\mathcal{V}$ is closed under products, quotients and subalgebras. (See Theorem 2.3.) We quickly discovered what at first we thought were extremely strange phenomena: although all infinitely generated free algebras always exist, sometimes initial algebras do not exist; sometimes homomorphisms cannot be decomposed into surjections followed by injections; sometimes equalizers do not exist; sometimes coequalizers do not exist.

All of these facts are explained by our Theorem 2.5: varieties of iteration theories have nice properties only when they coincide with a standard variety - i.e. only when the iteration operation $f \mapsto f \dagger$ is preserved by all theory congruences.

We assume the reader has some familiarity with theories and iteration theories (see [BEW80a, BEW80b, BÉ 88$]$ and the references there). This extended abstract contains no proofs. After a preliminary section which contains all of the necessary definitions, we give a detailed account of the results. Then we outline the main construction needed in the proofs of these results. This construction shows that for each iteration theory $T$, any $T$-algebra $\mathfrak{A}$ can be embedded in a $T$-iteration algebra $\mathfrak{A}^{\prime}$. In the last section, we indicate several kinds of examples of familiar and pathological varieties of iteration algebras. 


\section{Definitions: algebras and iteration algebras}

Let $T$ be an algebraic theory, not necessarily an iteration theory.

Definition $1.1 A T$-algebra $\mathfrak{A}=(A, \alpha)$ consists of a set $A$ and a theory morphism $\alpha: T \rightarrow$ Pow $_{A}$, where Pow $_{A}$ is the theory whose morphisms $n \rightarrow p$ are all functions $A^{p} \rightarrow A^{n}$ (see $[B W]$ ). The image $f \alpha$ of the $T$ morphism $f$ is usually written $f_{\alpha}$ or $f_{A}$.

Thus, in detail, the assignment $f \mapsto f_{\alpha}$ of functions to morphisms satisfies:

- $(f \cdot g)_{\alpha}=A^{p \stackrel{g_{\alpha}}{\rightarrow}} A^{n} \stackrel{f_{\alpha}}{\rightarrow} A^{m}$, all $f: m \rightarrow n, g: n \rightarrow p ;$

- $\langle f, g\rangle_{\alpha}=A^{n} \stackrel{\left(f_{\alpha}, g_{\alpha}\right\rangle}{\longrightarrow} A^{p+q}$, all $f: p \rightarrow n, g: q \rightarrow n$;

- $\left(i_{n}\right)_{\alpha}$ is the $i$ th projection $A^{n} \rightarrow A$.

The set $A$ is called the "underlying set" of the algebra $(A, \alpha)$. Note that if $f: n \rightarrow p$ is a $T$ morphism, the function $f_{\alpha}: A^{p} \rightarrow A^{n}$ is determined by the $n$ functions $g_{i}:=\left(i_{n} \cdot f\right)_{\alpha}: A^{p} \rightarrow A$ as follows:

$$
f_{\alpha}\left(a_{1}, \ldots, a_{p}\right)=\left(g_{1}\left(a_{1}, \ldots, a_{p}\right), \ldots, g_{n}\left(a_{1}, \ldots, a_{p}\right)\right) .
$$

Hence, we may specify a $T$-algebra by defining only the functions $f_{\alpha}$ for $f: 1 \rightarrow p$.

Definition 1.2 If $\mathfrak{A}=(A, \alpha)$ and $\mathfrak{B}=(B, \beta)$ are $T$-algebras, a homomorphism $h: \mathfrak{A} \rightarrow \mathfrak{B}$ is a function $h: A \rightarrow B$ such that for each $f: n \rightarrow p \in T$,

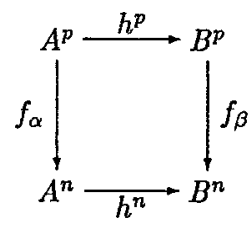

(Several equivalent definitions of $T$-algebras, as well as certain facts about the category of all categories of $T$-algebras, for all $T$, were given in [BW].)

DEFINITION 1.3 The collection of all T-algebras and homomorphisms determines a category, denoted $T^{b}$.

If $\mathfrak{A}$ and $\mathfrak{B}$ are $T$-algebras, we say that $\mathfrak{A}$ is a subalgebra of $\mathfrak{B}$, in symbols, $\mathfrak{A} \subseteq \mathfrak{B}$, if there is an injective homomorphism $h: \mathfrak{A} \rightarrow \mathfrak{B}$; we say that $\mathfrak{B}$ is a quotient or homomorphic image of $\mathfrak{A}$ if there is a surjective homomorphism $h: \mathfrak{A} \rightarrow \mathfrak{B}$. If $\mathfrak{A}_{i}=\left(A_{i}, \alpha_{i}\right), i \in I$, are $T$-algebras, the product

$$
\prod_{i \in I} \mathfrak{A}_{i}=\left(\prod_{i \in I} A_{i}, \alpha\right)
$$

is defined pointwise: for $f: 1 \rightarrow 2$, say, in $T$,

$$
f_{\alpha}\left(\left(a_{i}\right),\left(b_{i}\right)\right)=\left(f_{\alpha_{i}}\left(a_{i}, b_{i}\right)\right) .
$$

We note that if $\mathfrak{A}=(A, \alpha)$ is a $T$-algebra, then the relation $\sim_{\alpha}$ defined by

$$
f \sim_{\alpha} g \Leftrightarrow f_{\alpha}=g_{\alpha}
$$


is a theory congruence on $T$. Recall that a (theory) congruence on an algebraic theory $T$ is a family of equivalence relations $\theta_{n, p}$ on $T(n, p)$, for each pair $n, p \geq 0$, which are compatible with the theory operations of composition and tupling: if $f \theta_{n, p} g: n \rightarrow p$ and $f^{\prime} \theta_{p, q} g^{\prime}: p \rightarrow q$, then $\left(f \cdot f^{\prime}\right) \theta_{n, q}\left(g \cdot g^{\prime}\right)$ : $n \rightarrow q$; also if $f_{i} \theta_{1, p} g_{i}$, for each $i \in[n]$, then $\left\langle f_{1}, \ldots, f_{n}\right\rangle \theta_{n, p}\left\langle g_{1}, \ldots, g_{n}\right\rangle$. A theory congruence $\theta$ is trivial if $f \theta_{n, p} g$, for all $f, g: n \rightarrow p$, all $n, p$. A theory congruence on an iteration theory is a dagger congruence or iteration theory congruence, if $f \theta_{n, n+p} g \Rightarrow f^{\dagger} \theta_{n, p} g^{\dagger}$. From now on, we will write only $f \theta g$, and omit the subscripts on $\theta$.

REMARK 1.1 Let $\Omega$ be the signature (or ranked set) with $\Omega_{n}=T(1, n), n \geq 0$. Then an $\Omega$-algebra $A$ is a $T$-algebra iff $A$ satisfies all of the following equations:

$$
\begin{aligned}
h\left(x_{1}, \ldots, x_{n}\right) & =f\left(g_{1}\left(x_{1}, \ldots, x_{n}\right), \ldots, g_{p}\left(x_{1}, \ldots, x_{n}\right)\right) \\
i_{n}\left(x_{1}, \ldots, x_{n}\right) & =x_{i},
\end{aligned}
$$

for all $h=f \cdot\left\langle g_{1}, \ldots, g_{p}\right\rangle$ in $T$, where $f: 1 \rightarrow p$ and $g_{j}: 1 \rightarrow n$. Thus the class of all $T$-algebras is an equational class of $\Sigma$-algebras, where $\Sigma$ is the signature with $\Sigma_{n}=T(1, n), n \geq 0$.

Definition 1.4 Suppose that $T$ is an iteration theory. A $T$-iteration algebra is a $T$-algebra $\mathfrak{A}$ such that the relation $\sim_{\alpha}$ is a dagger congruence on $T$ - i.e. for all $f, g: n \rightarrow n+p$ in $T$,

$$
f \sim_{\alpha} g \Rightarrow f^{\dagger} \sim_{\alpha} g^{\dagger}
$$

Note that by virtue of the pairing identity (1), a $T$-algebra $\mathbb{A}=(A, \alpha)$ is a $T$-iteration algebra iff $f^{\dagger} \sim_{\alpha} g^{\dagger}$ whenever $f \sim_{\alpha} g$ for all $f, g: 1 \rightarrow 1+p$ in $T$.

REMARK 1.2 Let $\Omega$ be the same signature as in Remark 1.1. Then an $\Omega$-algebra $A$ is a $T$-iteration algebra iff $\mathfrak{A}$ is a $T$-algebra and also satisfies the following first order sentences:

$$
(\forall x)(\forall y)[f(x, y)=g(x, y)] \Rightarrow(\forall z)\left[f^{\dagger}(z)=g^{\dagger}(z)\right]
$$

for all $f, g: n \rightarrow n+p$ in $T$. Here, the variables $y$ and $z$ range over $A^{p}$ and $x$ ranges over $A^{n}$. Thus, the collection of all $T$-iteration algebras is first-order axiomatizable either by universal-existential or by existential-universal sentences.

Definition 1.5 We write $T^{\dagger}$ for the full subcategory of $T^{b}$ determined by the class of those $T$-algebras $\mathfrak{A}=(A, \alpha)$ such that $\sim_{\alpha}$ is a dagger congruence on $T$.

Both $T^{b}$ and $T^{\dagger}$ have an underlying set functor $U_{T}$ which maps the homomorphism $h: \mathfrak{A} \rightarrow \mathfrak{B}$ to the function $h: A \rightarrow B$.

If $\mathfrak{A}=(A, \alpha)$ is a $T$-iteration algebra, the collection $T \alpha$ of all functions $f_{\alpha}: A^{p} \rightarrow A^{n}$, for $f: n \rightarrow p$ in $T$ forms an iteration theory of functions, where the dagger operation on functions is defined by:

$$
\left(f_{\alpha}\right)^{\dagger}:=\left(f^{\dagger}\right)_{\alpha} \text {. }
$$

We call an iteration theory of functions a concrete iteration theory. Thus, a $T$-iteration algebra can be defined as a (surjective) iteration theory morphism from $T$ to a concrete iteration theory.

If $T$ is an iteration theory we abbreviate the morphism $1_{1}^{\dagger}: 1 \rightarrow 0$ by $\perp$. If $\mathfrak{A}=(A, \alpha)$ is any $T$-iteration algebra, we write $\perp_{\alpha}$ or $\perp_{A}$ for $\left(1_{1}{ }^{\dagger}\right)_{\alpha}$.

Suppose that $\sim$ is any dagger congruence on the iteration theory $T$. Write $R$ for the quotient theory $T / \sim$. Then, $\mathbb{A}=(A, \alpha) \in T^{\phi}$ determines an algebra in $R^{\dagger}$ iff the theory congruence $\sim_{\alpha}$ on $T$ is a dagger congruence, and $\sim \leq \sim_{\alpha}$. In this case, the theory morphism $\alpha: T \rightarrow$ Pow $_{A}$ factors uniquely through $R$. A case of some importance here is the case that $T$ is a free iteration theory. We recall from [BEW80a, BEW80b] that the theory $\Sigma t r$ of regular labeled trees is the iteration theory freely generated by the ranked set $\Sigma$. 


\section{Results}

There is a great deal known about the categories $T^{b}$. Indeed, these categories are the varieties of algebras (on a finitary signature). Thus, $T^{b}$ is complete, cocomplete, has surjective-injective factorizations, and has a free algebra generated by each set $X$ - i.e. the underlying set functor has a left adjoint. We are concerned mainly with the categories $T^{\dagger}$.

Definition 2.1 Suppose that $T$ is a fixed iteration theory. A full subcategory $\mathcal{V}$ of $T^{\dagger}$ is a variety of $T$-iteration algebras if

$$
\begin{aligned}
\mathcal{V} & =H(\mathcal{V}) \cap T^{\dagger} \\
& =S(\mathcal{V}) \cap T^{\dagger} \\
& =P(\mathcal{V}) \cap T^{\dagger}
\end{aligned}
$$

Here, $H(\mathcal{V})$ is the collection of all $T$-algebras which are (surjective) homomorphic images of algebras in $\mathcal{V} ; S(\mathcal{V})$ is the collection of all $T$-algebras which are subalgebras of algebras in $\mathcal{V} ; P(\mathcal{V})$ is the collection of all $T$-algebras which are products of algebras in $\mathcal{V}$.

If we delete the restriction " $\cap T^{\dagger "}$ "everywhere, we have the definition of a variety of $T$-algebras.

We state several of the main theorems.

THEOREM 2.1 For each infinite set $X$ there is an algebra in $T^{\dagger}$ freely generated by $X$.

As for the existence of finitely generated free algebras in $T^{\dagger}$ we have the following.

THEOREM $2.2 T^{\dagger}$ has an $n$-generated free algebra iff $\sim_{n}$ is a dagger congruence on $T$. Here,

$$
f \sim_{n} g \Leftrightarrow(\forall h: k \rightarrow n)(f \cdot h=g \cdot h),
$$

where $f, g: p \rightarrow k$ in $T$.

In all of the theorems below, we assume that $T$ is an iteration theory.

The varieties of $T$-iteration algebras are those categories $R^{\dagger}$, for some quotient iteration theory $R$ of $T$. More precisely, we have the following statement.

TH EOREM 2.3 Let $\mathcal{V}$ be a full subcategory of $T \dagger$. Then $\mathcal{V}$ is a variety of $T$-iteration algebras iff there is a dagger congruence $\theta$ on $T$ such that the $T$-iteration algebras $\mathfrak{A}=(A, \alpha)$ in $\mathcal{V}$ are exactly those for which $\alpha$ factors through the canonical map $T \rightarrow T / \theta$ :

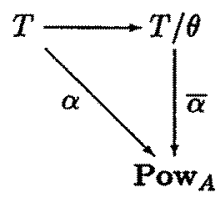

A special case of Theorem 2.3 may look more like a Birkhoff-type theorem. Suppose that $T=\Sigma t r$. Then $\mathcal{W}$ is a variety of $\Sigma t r$-iteration algebras iff there is a dagger congruence $\sim_{R}$ on $\Sigma t r$ such that $\mathcal{W}$ is isomorphic to $R^{\dagger}$, where $R=\Sigma t r / \sim_{R}$. 
Theorem 2.4 - Let $\mathcal{V}$ be a variety of $T$-algebras. Then $\mathcal{V} \cap T^{\dagger}$ is a variety of T-iteration algebras.

- Let $\mathcal{V}$ be a variety of $T$-iteration algebras. Then $H(\mathcal{V})$, the full subcategory of $T^{b}$ determined by all quotients of algebras in $\mathcal{V}$, is a variety of T-algebras. Moreover, $H(\mathcal{V})=S(\mathcal{V})$.

- The map from varieties of $T$-iteration algebras to varieties of $T$-algebras

$$
\mathcal{V} \mapsto H(\mathcal{V})
$$

is injective, but not always surjective.

It turns out that varieties of $T$-iteration algebras have nice properties only when every $T$-algebra is a $T$-iteration algebra.

ТнеоRем 2.5 The following statements are equivalent.

a. $T^{\dagger}=T^{b}$.

b. Every theory congruence on $T$ is a dagger congruence.

c. Every homomorphism $h: \mathfrak{A} \rightarrow \mathfrak{B}$ in $T^{\dagger}$ factors as

$$
h=\mathfrak{A} \stackrel{e}{\rightarrow} \mathfrak{C} \stackrel{m}{\rightarrow} \mathfrak{B}
$$

where $e$ is a coequalizer and $m$ is a monic.

d. Every homomorphism $h: \mathfrak{A} \rightarrow \mathfrak{B}$ in $T^{\dagger}$ factors as

$$
h=\mathfrak{A} \stackrel{\mathfrak{e}}{\rightarrow} \mathfrak{C} \stackrel{m}{\rightarrow} \mathfrak{B}
$$

where $e$ is an epi and $m$ is a monic.

e. $T^{\dagger}$ has equalizers.

f. $T^{\dagger}$ is complete.

g. $T^{\dagger}$ has coequalizers.

h. $T^{\dagger}$ is cocomplete.

i. The inclusion functor $T^{\dagger} \hookrightarrow T^{\phi}$ has a left adjoint.

j. For each $\mathfrak{A} \in T^{\mathfrak{b}}$, and $\mathfrak{B} \in T^{\dagger}$ with $\mathfrak{A}$ a subalgebra of $\mathfrak{B}$, there is a least subalgebra $\mathfrak{C} \subseteq \mathfrak{B}$ in $T^{\dagger}$ with $\mathfrak{A} \subseteq \mathfrak{C}$.

\section{The Embedding Theorem}

The main tool in the proof of Theorem 2.5 is the Embedding Theorem. This latter result shows that for any iteration theory $T$ and any $T$-algebra $\mathfrak{A}$, there is a $T$-iteration algebra $\mathfrak{B}$ and an injective homomorphism $\mu: \mathfrak{A} \rightarrow \mathfrak{B}$. In order to prove the extension theorem, we found it necessary to find an explicit representation of the coproduct $T[X]$ of the iteration theories $T$ and $\left[X, x_{0}\right]$, the iteration theory freely generated by the set $X$ of morphisms $1 \rightarrow 0$. The iteration theory $T[X]$ has the property that there are iteration theory morphisms

$$
T \stackrel{\kappa}{\rightarrow} T[X] \stackrel{\lambda}{\leftarrow}\left[X, x_{0}\right]
$$


such that $x_{0} \lambda=1_{1}^{\dagger}$, with the following universal property. For any iteration theory $R$ and any iteration theory morphism $\varphi: T \rightarrow R$ and any function $\psi: X \rightarrow R(1,0)$ there is a unique iteration theory morphism $\langle\varphi, \psi\rangle: T[X] \rightarrow R$ with $\kappa \cdot\langle\varphi, \psi\rangle=\varphi$ and $\lambda \cdot\langle\varphi, \psi\rangle=\psi$.

Using this description, for any $T$-algebra $\mathfrak{A}$ in $T^{b}$, we show how to construct an iteration theory $T(\mathfrak{A})$. This theory has the property that the initial algebra in $T(\mathfrak{A})^{\mathfrak{b}}$ is $\mathfrak{A}$ with all elements in $A$ adjoined as constants; more generally, the $T(\mathfrak{A})$-algebras are those $T$-algebras $\mathfrak{B}$ for which there is some homomorphism $\mathfrak{A} \rightarrow \mathfrak{B}$. If we now form the theory $T^{\prime}=T(\mathfrak{A})[X]$, for an infinite set $X$, it turns out that the $T^{\prime}$-morphisms $1 \rightarrow 0$ determine a $T$-iteration algebra containing an isomorphic copy of $\mathfrak{a}$.

\section{Examples}

EXAMPLE 4.1 We give an example of an iteration theory $T$ for which there is no initial algebra in $T^{\dagger}$. Let $\Sigma_{1}=\{f\} ; \Sigma_{0}=\{1\}$ and $\Sigma_{n}=\emptyset$ otherwise. We consider the theory $T$ which is the quotient of $\Sigma t r$ by the least theory congruence $\sim$ such that

$$
f \cdot \perp \sim \perp
$$

where of course, $\perp=1_{1}^{\dagger} . T$ is an iteration theory. Each tree $1 \rightarrow 0$ in $\Sigma t r$ is equivalent to either $\perp$ or to $f^{\dagger}$. The morphisms $1 \rightarrow p$ in $T$ are the trees $f^{k} \cdot i_{p}$, for some $k \geq 0$ and $i \in[p]$, or $f^{\dagger} \cdot 0_{p}$ or $\perp \cdot 0_{p}$.

In $T, \sim_{0}$ is a congruence which is not a dagger congruence, since, writing $[t]$ for the $\sim$-congruence class of $t$,

$$
[f] \sim_{0}\left[\mathbf{1}_{1}\right]
$$

but

$$
\left[f^{\dagger}\right] \varkappa_{0}[\perp]
$$

EXAm Ple 4.2 For any set $A$, every theory congruence on $\operatorname{Pfn}_{A}$ is a dagger congruence. In fact, we can describe all theory congruences on $\operatorname{Pfn}_{A}$ as follows. For any two partial functions $f, g: A \times[n] \rightarrow$ $A \times[p]$, let

$$
D(f, g):=\left\{(a, i) \in A \times[p]: f^{-1}(a, i) \neq g^{-1}(a, i)\right\} .
$$

Let $\kappa$ be an infinite cardinal. Define

$$
f \sim_{\kappa} g \Leftrightarrow|D(f, g)|<\kappa .
$$

Then, for infinite sets $A$, the lattice of theory congruences on $\mathbf{P f n}_{A}$ is a well-ordered chain

$$
=A, \sim \mathcal{K}_{0}, \ldots, \sim_{|A|}, \sim_{|A|+}
$$

with least element the identity congruence, and greatest element the trivial congruence $\sim|A|^{+}$. On finite sets with at least two elements, there are exactly two theory congruences on $\operatorname{Pfn}_{A}$ : the identity and the trivial congruence.

EXAMPLE 4.3 An example of an iteration theory $T$ such that $T^{\dagger}$ has all free algebras, but $T^{\dagger} \neq T^{b}$. If $T=M^{2} t_{S}$ is a matrix theory, i.e. the morphisms $n \rightarrow p$ in $T$ are $n$ by $p$ matrices over a semiring $S$, then a $T$-algebra is essentially an $S$-module. In the case that $T$ is a matrix iteration theory, for each element $s \in S$, in $T$ there is an element $s^{*} \in S$ satisfying certain equational properties (see [BÉa, BÉb]). For example,

$$
s^{*}=s s^{*}+1,
$$


for all $s \in S$, where $1=1_{1}$. Thus, for any element $a$ in the $S$ module $A$, and any $s \in S$,

$$
s^{*} a=a+s a+s^{2} a+\ldots+s^{n} a+s^{n+1} s^{*} a,
$$

for each $n \geq 0$. An $S$-module $A$ is a $T$-iteration algebra iff for each pair $s, t \in S$, the following implication holds:

$$
(\forall a \in A)(s a=t a) \Rightarrow(\forall a \in A)\left(s^{*} a=t^{*} a\right) .
$$

It is easy to see that for matrix theories, each theory congruence $\sim_{n}$ (defined above in (2)) is the identity congruence when $n>0$; $\sim_{0}$ is the trivial congruence. In any case, each congruence $\sim_{n}$ is a dagger congruence, so that $T^{\dagger}$ has all finitely generated free algebras. It follows that $T^{\dagger}$ has all free algebras, by Theorem 2.1 .

However, not every theory congruence on matrix iteration theories is a dagger congruence. For one example, consider the semiring $S$ of all subsets of words on an alphabet $X$ containing at least two distinct letters. Any relation $\sim$ on sets of words extends in the obvious way to matrices over $S: A \sim B$ iff $A_{i j} \sim B_{i j}$, for all $i, j$. Now for any fixed nonnegative integer $n$, we define $w={ }_{n} w^{\prime}$, if the length of $w$ is the same as the length of $w^{\prime}$ and it is possible to change up to $n$ letters in $w$ to obtain $w^{\prime}$.

For sets of words $A, B$, say that $A<_{n} B$ if for each word $w$ in $A$ there is a word $w^{\prime}$ in $B$ with $w={ }_{n} w^{\prime}$. Now define

$$
A \sim B \Leftrightarrow(\exists n)\left(A<_{n} B \wedge B<_{n} A\right) .
$$

Then, $\sim$ determines a theory congruence on $M a t_{S}$ which is not a dagger congruence.

EXAMPLE 4.4 If $Z$ is any subset of natural numbers, there is a variety $\mathcal{V}_{Z}$ of iteration algebras having an $n$-generated free iteration algebra iff $n \in Z$. In particular, there is an iteration theory $T$ such that $T^{\dagger}$ has no finitely generated free algebras.

EXAMPLE 4.5 For any fixed signature $\Sigma$, we exhibit a subclass of algebras in $(\Sigma t r)^{\dagger}$. Suppose that $(A,<)$ is an $\omega$-complete poset, with least element $\perp$. Let $f$ be any $\omega$-continuous and monotone function

$$
f: A^{n+p} \rightarrow A^{n}
$$

Let $y$ be some element in $A^{p}$. The sequence of elements in $A^{n}$ :

$$
b_{0}=(\perp, \ldots, \perp), b_{1}=f\left(b_{0}, y\right), \ldots, b_{k+1}=f\left(b_{k}, y\right), \ldots
$$

is increasing and has a least upper bound, denoted $f^{\dagger}(y)$. It is not hard to show that the function

$$
\begin{gathered}
A^{p} \rightarrow A^{n} \\
y \mapsto f^{\dagger}(y)
\end{gathered}
$$

is also $\omega$-continuous and monotone. Further, for all $y \in A^{p}$,

$$
f^{\dagger}(y)=f\left(f^{\dagger}(y), y\right) \text {. }
$$

Let $\operatorname{Pow}_{\omega}(A)$ denote the subtheory of $\operatorname{Pow}_{A}$ whose morphisms are the $\omega$-continuous, monotone functions. Then, with the above definition of the iteration operation, $\operatorname{Pow}_{\omega}(A)$ is an iteration theory [BÉ89]. Hence, if $\alpha$ is a function which assigns to each symbol in $\Sigma$ a function in $P_{o w}(A), \alpha$ extends uniquely to an iteration theory morphism $\Sigma t r \rightarrow \operatorname{Pow}_{\omega}(A)$.

Thus, all $\omega$-continuous algebras are iteration algebras.

Similarly, one can show that the regular algebras of Tiuryn [Tiu78] are iteration algebras. 
EXAMPLE 4.6 For any signature $\Sigma$, an iterative $\Sigma$-algebra ([Nel81, Tiu80]) is a $\Sigma$-algebra $\mathfrak{A}$ with the property that for each $n$-tuple of nontrivial polynomial functions $p_{i}(\bar{x}, \bar{y}), i \in[n]$, in $n+p$ variables $\bar{x}=\left(x_{1}, \ldots, x_{n}\right)$ and $\bar{y}=\left(y_{1}, \ldots, y_{p}\right)$, for each choice of $p$ elements $b_{1}, \ldots, b_{p}$ in $A$, there is a unique solution to the system of equations

$$
\begin{aligned}
x_{1} & =p_{1}(\bar{x}, \bar{b}) \\
& \vdots \\
x_{n} & =p_{n}(\bar{x}, \bar{b}) .
\end{aligned}
$$

An iterative algebra may be extended to an iteration algebra by making a choice of a distinguished element $\perp$ in $A$ (see [BEW80a]).

REMARK 4.1 Every variety of iteration algebras generated by a class of $\omega$-continuous algebras has all free algebras. The same is true for the varieties generated by iterative algebras.

EXAMPLE 4.7 If $T$ is the iteration theory of regular synchronization trees over a set of action symbols ([BÉT]), all free algebras exist in $T^{\dagger}$. This fact can be established by showing that each of the congruences $\sim_{n}$ is the identity congruence in this theory.

EXAMPLE 4.8 A $T$-iteration algebra $\mathfrak{A}=(A, \alpha)$ is strong if the following sentences are true in $\mathfrak{A}$ :

$$
\forall y \in A^{p}\left[\forall x \in A^{n}[f(x, y)=g(x, y)] \Rightarrow f^{\dagger}(y)=g^{\dagger}(y)\right]
$$

for each pair $f, g: n \rightarrow n+p$ in $T$. These algebras were introduced in [Ési83]. We mention here the fact that there are iteration theories $T$ having a $T$-iteration algebra which is not strong. But every $\omega$-continuous algebra and iterative algebra is a strong iteration algebra.

\section{References}

[BÉa] S.L. Bloom and Zoltán Ésik. Matrix and matricial iteration theories, part I. To appear. [BÉb] S.L. Bloom and Zoltán Ésik. Matrix and matricial iteration theories, part II. To appear.

[BÉ88] S.L. Bloom and Z. Ésik. Varieties of iteration theories. SIAM Journal of Computing, $17(5): 939-966,1988$.

[BÉ89] S.L. Bloom and Z. Ésik. Equational logic of circular data type specification. Theoretical Computer Science, 63(3):303-331, 1989.

[BÉT] S.L. Bloom, Z. Ésik, and D. Taubner. Iteration theories of synchronization trees. Information and Computation, to appear.

[BEW80a] S.L. Bloom, C.C. Elgot, and J.B. Wright. Solutions of the iteration equation and extensions of the scalar iteration operation. SIAM Journal of Computing, 9(1):26-45, 1980.

[BEW80b] S.L. Bloom, C.C. Elgot, and J.B. Wright. Vector iteration in pointed iterative theories. SIAM Journal of Computing, 9(3):525-540, 1980.

[BW] S.L. Bloom and E.G. Wagner. Many sorted theories and their algebras. In Algebraic Methods in Semantics. Cambridge University Press.

[CG87] V.E. Cazanescu and Gh. Ştefănescu. Towards a new algebraic foundation of flowchart system theory. Research Report 43, Institute of Mathematics, Institute for Scientific and Technical Creation, Bucharest, Romania, 1987. 
[CN76] B. Courcelle and M. Nivat. Algebraic families of interpretations. In 17th IEEE Symp. on Foundations of Computing, 1976.

[Ési80] Z. Ésik. Identitites in iterative and rational theories. Computational Linguistics and Computer Languages, 14:183-207, 1980.

[Ési83] Z. Ésik. Algebras of iteration theories. Journal of Computer and System Science, $27(2): 291-303,1983$.

[Ési90] Z. Ésik. A note on the axiomatization of iteration theories. Acta Cybernetica, 1990. To appear.

[GTWW77] J. Goguen, J. Thatcher, E. Wagner, and J. Wright. Initial algebra semantics and continuous algebras. Journal of the Association of Computing Machinery, (24):68-95, 1977.

[Guess81] I. Guessarian Algebraic Semantics. Lecture Notes in Computer Science, 99, 1981.

[HS73] H. Herrlich and G.E. Strecker. Category Theory. Allyn and Bacon, 1973.

[Law63] F.W. Lawvere. Functorial semantics of algebraic theories. Proceedings of the National Academy of Sciences USA, 50:869-873, 1963.

[Ne181] Evelyn Nelson. Iterative algebras. Technical report, McMaster University, 1981.

[Niv75] M. Nivat. On the interpretation of recursive polyadic program schemes. Symposia Mathematica, 15:255-281, 1975.

[Stef87a] G. Stefănescu. On flowchart theories: Part I. The deterministic case. Journal of Computer and System Science, (35):163-191, 1987.

[Stef87b] G. Stefănescu. On flowchart theories: Part II. The nondeterministic case. Theoretical Computer Science, (52):307-340, 1987.

[Tiu78] Jerzy Tiuryn. Fixed points and algebras with infinitely long expressions, I. regular algebras. Fundamenta Informatica, 2(1):103-127, 1978.

[Tiu80] Jerzy Tiuryn. Unique fixed points vs. least fixed points. Theoretical Computer Science, 12(3):229-254, 1980.

[Wag90] E.G. Wagner. Algebras, polynomials and programs. Theoretical Computer Science, 70:3$34,1990$.

[WTGW76] J.B. Wright, J.W. Thatcher, J. Goguen, and E.G. Wagner. Rational algebraic theories and fixed-point solutions. In Proceedings 17th IEEE Symposium on Foundations of Computing, pages 147-158, Houston, Texas, 1976. 